\title{
CONCEPÇÃo de PROFISSIONAIS DE SAÚde QUE ATUAM EM EMERGÊNCIA DE SAÚDE MENTAL
}

\section{Marcos Hirata Soares ${ }^{1}$ Eloisi Delalibera Ruzzon ${ }^{2}$ Maira Sayuri Sakay Bortoletto ${ }^{3}$}

O presente estudo comparou as concepções sobre doença mental de profissionais administrativos, de enfermagem e médicos dos serviços de saúde de urgência no município de Londrina-PR. O delineamento foi transversal, utilizando a escala OpinionsAbout Mental Illness (OMI) com uma amostra aleatória de 30\% dos profissionais, totalizando 96 sujeitos no período de 2010 a 2011. Os dados foram submetidos à análise estatística pelo StatisticalPackage for Social Sciences (SPSS). Para dois fatores da escala ODM e com os enfermeiros, há normalidade na distribuição amostral. O teste de Kruskall-Wallis indicou que há diferença significativa com relação a quatro dos sete fatores componentes da ODM $(\mathrm{p}<0,05)$. Os profissionais que atuam nesses serviços necessitam de capacitação na área de saúde mental, bem como há a necessidade de novos estudos sobre o tema, com um maior número de sujeitos.

Descritores: Transtornos Mentais; Serviços Médicos de Emergência; Enfermagem Psiquiátrica; Serviços de Emergência Psiquiátrica.

\footnotetext{
${ }^{1}$ Doutorando, Escola de Enfermagem de Ribeirão Preto, Universidade de São Paulo, Centro Colaborador da OMS para o Desenvolvimento da Pesquisa em Enfermagem, Ribeirão Preto, SP, Brasil.

2 Aluna, Programa de Residência Multiprofissional em Saúde da Mulher. Universidade Estadual de Londrina, Londrina, PR, Brasil.

${ }^{3}$ Enfermeira, Departamento de Saúde Coletiva, Universidade Estadual de Londrina, Londrina, PR, Brasil.
} 


\section{CONCEPTION OF HEALTH PROFESSIONALS WHO WORK IN EMERGENCY MENTAL}

\section{HEALTH}

This study compared the conceptions about mental illness of administrative professionals, nurses and doctors of emergency health services in the city of Londrina, PR. The design was cross-sectional, using the Opinions about Mental Illness (OMI) scale with a random sample of $30 \%$ of the professionals, totaling 96 subjects in the period 2010 to 2011 . The data was statistically analyzed by the Statistical Package for Social Sciences (SPSS); with two factors of the OMI scale and nurses, there was normality in the sampling distribution. The Kruskall-Wallis test indicated a significant variation with respect to four of the seven factor components of the OMI ( $\mathrm{p}<0.05$ ). The professionals that work in emergency health services require training in the area of mental health, and there is a need for further studies on this topic, with a larger number of subjects.

Descriptors: Mental Disorders; Emergency Medical Services; Psychiatric Nursing; Emergency Services, Psychiatric.

\section{CONCEPCIÓN DE PROFESIONALES DE LA SALUD QUE ACTÚAN EN URGENCIAS DE LOS SERVICIOS DE SALUD MENTAL}

El presente estudio comparó las concepciones sobre enfermedad mental de profesionales administrativos, de enfermería y médicos de los servicios de salud de urgencias en el municipio de Londrina-PR. O delineamiento fue transversal, utilizando la escala OpinionsAbout Mental Illness (OMI) con una muestra aleatoria del 30\% de los profesionales, totalizando 96 sujetos en el período de 2010 a 2011. Los datos fueron sometidos al análisis estadístico por el StatisticalPackage for Social Sciences (SPSS). Para dos factores de la escala ODM y con los enfermeros, hay normalidad en la distribución muestral. El test de Kruskall-Wallis indicó que hay diferencia significativa con relación a cuatro de los siete factores componentes de la ODM $(\mathrm{p}<0,05)$. Los profesionales que actúan en esos servicios necesitan de capacitación en el área de salud mental, al igual que existe la necesidad de nuevos estudios sobre el tema, con un mayor número de sujetos.

Descriptores: Trastornos Mentales; Servicios Médicos de Urgencia; Enfermería Psiquiátrica; Servicios de Urgencia Psiquiátrica.

\section{Introdução}

A reforma psiquiátrica no Brasil é um movimento histórico político, social e econômico que vêm contribuindo para a prestação de uma assistência aos portadores de transtornos mentais não mais exclusivamente nos hospitais psiquiátricos, e sim nos Centros de Atenção Psicossocial
(CAPS) e demais serviços da rede de cuidados em saúde mental, como Unidades de Saúde da Família (USF) e Serviços de Urgência, por exemplo, auxiliando na conquista de mais cidadania para esses indivíduos e contribuindo para a desmistificação do doente e da doença mental ${ }^{(1)}$.

Nos atendimentos em situações de emergência psiquiátrica encontra-se muitas vezes a 
preocupação exclusiva com os aspectos biológicos do cuidar, como a administração de medicamentos e cuidados de higiene, por exemplo ${ }^{(2)}$, visando a preservação da vida sem que os aspectos psíquicos sejam muitas vezes considerados, não caracterizando uma assistência integral em saúde mental.

Um estudo realizado na cidade de São Paulo constatou que $70 \%$ dos pacientes com primeiro episódio psicótico deram entrada no sistema de saúde por meio do serviço de emergência, e 50\% dos casos foi feito em até quatro semanas do início dos sintomas ${ }^{(3)}$.

É comum que estudos nesta temática não envolvam profissionais diversos, como os de apoio administrativo, por exemplo. Neste estudo, houve a iniciativa de incluir outros profissionais além da enfermagem, justamente pelo seu contato direto ou indireto com esses clientes, demandando também a necessidade de capacitação para conseguirem melhor lidar com esse público, pois um dos grandes desafios na Reforma Psiquiátrica é resgatar a cidadania do doente mental e a diminuição do estigma e preconceito em relação à doença mental ${ }^{(4-5)}$.

Dentre os diversos profissionais de saúde que atuam em saúde mental, o enfermeiro e sua equipe são quem mais mantêm contato com os usuários. No entanto, o enfermeiro muitas vezes apresenta uma atuação com o paciente de forma autoritária de controlar, vigiar e reprimir o mesmo, no qual a melhor forma de agir é com atitudes terapêuticas diante do cliente em sofrimento psíquico, como, por exemplo, o acolhimento e empatia, assim como ter bom conhecimento sobre psicofarmacologia, psicopatologia, alicerçados nos princípios da Reforma Psiquiátrica e Reabilitação Psicossocial.

Há na literatura a referência aos estudos "Atitudes frente à doença mental: estudo comparativo entre ingressantes e formandos em enfermagem", no qual existem mudanças de comportamento nos acadêmicos em relação ao paciente mental, quanto maior nível de escolaridade ${ }^{(6)}$, e "Atitudes de empresários do Sul do Brasil em relação aos portadores de doenças mentais", no qual os administradores apresentam uma visão protecionista, de irrecuperabilidade e periculosidade, possivelmente devido à desinformação e ao preconceito $^{(7)}$.
Um estudo $^{(8)}$ feito na Grécia com 239 estudantes de medicina e 361 funcionários diversos constatou que os profissionais da ala psiquiátrica tiveram atitudes mais positivas em relação a pacientes com doença mental. Os autores concluíram que o contato com pacientes portadores de doença mental e a formação são importantes na redução do estigma entre o pessoal de saúde mental e de outros setores. Contudo, não foram encontrados estudos envolvendo profissionais como médicos e auxiliares administrativos no Brasil, bem como em serviços de urgência.

Com o intuito de avaliar a opinião sobre a doença mental em profissionais de serviços de urgência e emergência, foi utilizada a escala de ODM (Opiniões sobre a Doença Mental), validada no Brasil ${ }^{(9)}$ para avaliar a concepção sobre doença mental em profissionais administrativos, de enfermagem e médicos que atuam em LondrinaPR, uma vez que não se dispõem de tal informação, o que torna o estudo pioneiro neste tema. Assim, a hipótese para este estudo é que haja diferenças nas concepções frente ao portador de transtorno mental, mas restando descrever como ou quais são tais diferenças e se outras variáveis estariam associadas a esse processo. Torna-se importante descrever tais concepções, uma vez que a assistência prestada ao paciente é de maior qualidade, quando mais categorias profissionais envolvidas nesse cuidado forem qualificadas por meio da identificação das concepções de doença mental com os sujeitos deste estudo.

\section{Objetivo}

Comparar a concepção sobre doença mental entre profissionais administrativos, de enfermagem e médicos dos serviços públicos de urgência e emergência em saúde no município de Londrina-PR.

\section{Metodologia}

Trata-se de um estudo transversal individuado, com análise de 96 profissionais de diversos serviços de urgência e emergência médica de Londrina, entre eles o Hospital Universitário regional do Norte do Paraná, o Serviço de Atendimento Móvel de urgência (SAMU), o Centro de Atenção Psicossocial (CAPS) e dois Hospitais estaduais secundários. 
Sujeitos e local

Os dados foram coletados em momento adequado onde não houvesse empecilho durante as jornadas de trabalho e no melhor horário para os sujeitos que aceitaram participar do estudo. A amostra da pesquisa foi de $30 \%$ dos profissionais do serviço de urgência e emergência, representados por enfermeiros, técnicos e auxiliares de enfermagem*, médicos e profissionais do setor de administrativo, obtendo um total de 96 (90,5\%do total da amostra) de entrevistados de ambos os gêneros.

O critério de inclusão como sujeito na pesquisa foi feito por meio de amostragem aleatória simples, sendo participantes somente profissionais do serviço de emergência, ou seja, os prontos-socorros dos diversos locais, baseando-se num percentual de $30 \%$ do quadro de profissionais de cada serviço e categoria, bem como foi realizada primeiramente a escolha pelos diversos turnos de trabalho, seguida de um sorteio que permitiu uma abordagem dos entrevistados para participação.

Caso o profissional recusasse, era realizado um novo sorteio. Se por algum motivo o sujeito não respondesse à escala ODM, ela era novamente entregue ao participante, até no máximo três vezes. Assim, se por ventura o profissional não aceitasse, era novamente sorteado outro indivíduo.

Devido à dinâmica dos serviços de urgência e emergência, houve a impossibilidade de participação da pesquisa de oito técnicos de enfermagem e de dois médicos, fazendo que outros fossem sorteados em seu lugar; a coleta de dados foi realizada nos meses de novembro e dezembro de 2010 e de janeiro a abril de 2011.

Instrumento de coleta de dados

Utilizou-se como instrumento, um questionário autoaplicável, composto de duas partes. A primeira com informações como idade, sexo, profissão, experiência profissional e escolaridade, e a segunda com uma escala de atitudes -
“Opinions about Mental Illness". Essa escala é representada no Brasil pelas iniciais ODM (Opiniões sobre a Doença Mental), assim denominada quando traduzida e validada para o idioma português ${ }^{(10-11)}$.

Em sua versão latina, a escala é composta por sete fatores para análise e por 51 questões, cada uma com seis alternativas do tipo Likert, seguindo a sequência progressiva de seis pontos de concordância, desde "concordo totalmente" até "discordo totalmente". Quanto maior o escore obtido, maior a crença da pessoa à ideia contida em cada fator. Quanto menor o escore, menor a crença. A escala possui sete fatores descritos abaixo:

- Autoritarismo. Reflete a perspectiva de que o doente mental necessita ser isolado de outros pacientes, permanecendo sob portas trancadas e vigilância. Contém o conceito da irrecuperabilidade pessoal e social do doente e a ideia de sua periculosidade. Neste fator estão incluídas nove afirmações.

- Benevolência. Traduz a visão de que o doente mental, devido à sua infelicidade, deve ser amparado por meio de protecionismo bondoso e paternalista, com base em cuidados, atenção pessoal e conforto material. Neste fator estão incluídas cinco afirmações.

- Ideologia da higiene mental. Representa a ideia de que o doente mental é uma pessoa semelhante às pessoas normais, com diferenças quantitativas, porém não qualitativas. Podem desempenhar atividades especializadas e até cuidar de crianças. Neste fator estão incluídas onze afirmações.

- Restrição social. Traduz a doença mental como uma espécie de defeito hereditário, completamente diferente de outras doenças, cujo portador pode contaminar

\footnotetext{
* No Brasil, o técnico de enfermagem é o profissional que possui o nível médio de escolaridade e executa procedimentos técnicos sob supervisão do enfermeiro.
} 
a família e a sociedade, devendo, portanto, ser protegidas por meio de restrição dos direitos pessoais e sociais do paciente, mesmo após a hospitalização. Neste fator estão incluídas sete afirmações.

- Etiologia interpessoal. Explica a doença mental como originária de vivências interpessoais, com ênfase para a interação com figuras parentais. Neste fator estão incluídas seis afirmações.

- Etiologia de esforço mental. Reflete a ideia de que a doença mental se origina de "excessivo esforço cerebral" pelo trabalho intelectual exagerado, por pensar demais ou por ter pensamentos negativos. Neste fator estão incluídas oito afirmações.

- Visão minoritária. Traduz o conceito de que o doente mental, por ser muito diferente das pessoas consideradas normais, pode ser facilmente reconhecido em um agrupamento humano, principalmente pela sua aparência externa. Neste fator estão incluídas cinco afirmações.

\section{Análise de dados}

Os escores fatoriais brutos obtidos com a aplicação da ODM foram transformados para o sistema $\operatorname{Sten}^{(11-12)}$, que proporciona uma comparação padrão entre os fatores com variação entre 1 e 10 , média de 5,5 e um desvio padrão igual a 0,5 . Foi criado um banco de dados que foi analisado pelo programa SPSS- v.15. O passo inicial foi realizar um teste de normalidade, visando escolher entre testes paramétricos, caso a distribuição fosse normal e não paramétricos, caso ela fosse não normal. $\mathrm{O}$ nível de significância utilizado foi de $\alpha=0.05$.

\section{Procedimento}

Inicialmente, foi feito contato com as instituições envolvidas no estudo para a devida autorização, com a apresentação da pesquisa submetida ao Comitê de Ética em Pesquisa Envolvendo
Seres Humanos da UEL/HURNP (CAAE $n^{घ}$ 3060.0.000.268-09) e também da Autarquia Municipal de Saúde de Londrina para a coleta de dados nos serviços públicos de emergência médica de Londrina-PR. Assim, foram sorteados $30 \%$ de cada classe, sendo enfermeiros, técnicos de enfermagem, médicos e auxiliares administrativos. Todos os participantes assinaram o termo de consentimento livre e esclarecido como sujeitos da pesquisa.

\section{Resultados}

O primeiro passo para a análise estatística foi realizar o teste de normalidade Kolmogorovsmirnov e Shapiro-Wilk. Esses testes indicaram que apenas os fatores Ideologia de Higiene Mental e Visão Minoritária possuem distribuição normal $(\mathrm{p}>0,05)$ e apenas para a profissão de Enfermeiro. Para os demais fatores e profissões, todos possuem distribuição não normal $(\mathrm{p}<0,05)$. Assim, optou-se por caracterizar a amostra como de distribuição não normal e utilizou-se o teste não paramétrico de KruskallWallis para comparar se há significância estatística na diferenças entre as medianas das quatro profissões.

Participaram da pesquisa 96 profissionais, sendo 17 enfermeiros (14,2\%), 57 técnicos de enfermagem (47,5\%), 14 médicos $(11,7 \%)$, e 8 auxiliares administrativos (6,7\%). Quanto ao gênero, $54,1 \%$ do sexo feminino e $45,9 \%$ do sexo masculino. A idade variou dos 20 a 66 anos, obtendo uma média de 24 anos. Os profissionais em maior número foram os técnicos de enfermagem com 57 entrevistados, correspondendo a 47,5\% , seguidos dos enfermeiros com 17 sujeitos da pesquisa correspondendo a $14,2 \%$ dos entrevistados, 14 médicos e 8 auxiliares administrativos, correspondendo a $11,7 \%$ e $6,7 \%$, respectivamente. Esse dado já era esperado, uma vez que a grande parte dos profissionais da equipe de serviços de saúde em sua maioria é representada pela enfermagem.

Quanto à escolaridade, a maior porcentagem foi a de nível técnico (22,5\%), seguida de superior completo (20\%), especialização $(19,2 \%)$ e superior incompleto $(16,7 \%)$. Quanto ao local de estudo, foram entrevistados $29(24,2 \%)$ profissionais no Hospital 
Universitário devido ao maior número de funcionários existente neste ambiente de traba1ho, seguido de um Hospital Estadual com 22 $(18,3 \%)$ participantes, $19(15,8 \%)$ no SAMU e $6(5,0 \%)$ no Centro de Apoio Psicossocial (CAPS 111). Quanto à experiência profissional, houve um número de 54 (45\%) de trabalhadores de 1 a 10 anos de profissão, de 11 a 20 anos $29(24,2 \%)$ e $13(10,80 \%)$ com mais de 20 anos de profissão.

As médias, medianas e desvio padrão para os escores dos fatores da escala ODM foram apresentados na Tabela 1 .

Tabela 1 - Média, mediana e desvio padrão dos escores da escala ODM para auxiliares administrativos, profissionais de enfermagem e médicos, Londrina-PR, Brasil, 2010-2011

\begin{tabular}{|c|c|c|c|}
\hline & Média & Mediana & $\begin{array}{l}\text { Desvio } \\
\text { padrão }\end{array}$ \\
\hline \multicolumn{4}{|l|}{ Autoritarismo } \\
\hline Auxiliar administrativo & 8,75 & 9,0 & ,24 \\
\hline Enfermeiro & 9,94 & 10,00 &, 24 \\
\hline Médico & 9,93 & 10,00 & ,26 \\
\hline Técnico em Enfermagem & 9,89 & 10,00 & ,45 \\
\hline Auxiliar administrativo & 3,13 & 2,5 & 1,45 \\
\hline \multicolumn{4}{|l|}{ Benevolência } \\
\hline Enfermeiro & 1,24 & 1,00 & ,43 \\
\hline Médico & 1,21 & 1,00 & ,42 \\
\hline Técnico em Enfermagem & 1,21 & 1,00 & ,90 \\
\hline \multicolumn{4}{|l|}{ Ideologia de higiene mental } \\
\hline Auxiliar administrativo & 3,13 & 2,50 & 1,45 \\
\hline Enfermeiro & 3,82 & 4,00 & 1,81 \\
\hline Médico & 2,79 & 3,00 & 1,12 \\
\hline Técnico em Enfermagem & 4,32 & 4,00 & 1,76 \\
\hline \multicolumn{4}{|l|}{ Restrição social } \\
\hline Auxiliar administrativo & 8,75 & 8,00 & 1,03 \\
\hline Enfermeiro & 9,94 & 10,00 & ,243 \\
\hline Médico & 9,86 & 10,00 & ,363 \\
\hline Técnico em Enfermagem & 9,82 & 10,00 & 428 \\
\hline Auxiliar administrativo & 7,88 & 7,00 & 1,246 \\
\hline \multicolumn{4}{|l|}{ Etiologia Interpessoal } \\
\hline Enfermeiro & 8,82 & 10,00 & 1,629 \\
\hline Médico & 9,71 & 10,00 & ,469 \\
\hline Técnico em Enfermagem & 8,28 & 8,00 & 1,656 \\
\hline \multicolumn{4}{|l|}{ Etiologia de esforço mental } \\
\hline Auxiliar administrativo & 8,75 & 8,00 & 1,035 \\
\hline Enfermeiro & 9,94 & 10,00 & ,243 \\
\hline Médico & 9,86 & 10,00 & ,363 \\
\hline Técnico em Enfermagem & 9,28 & 10,00 & ,921 \\
\hline \multicolumn{4}{|l|}{ Visão minoritária } \\
\hline Auxiliar administrativo & 6,25 & 6,00 & 1,488 \\
\hline Enfermeiro & 7,24 & 7,00 & 1,640 \\
\hline Médico & 8,79 & 9,00 & 1,311 \\
\hline Técnico em Enfermagem & 6,61 & 6,00 & 1,677 \\
\hline
\end{tabular}

Tabela 2 - Comparação entre as categorias profissionais em relação aos fatores da escala ODM, pelo Teste de Kruskall-Wallis, Londrina, PR, Brasil, 2011

\begin{tabular}{lc}
\hline & Sig. assintótica \\
\hline Autoritarismo &, 894 \\
Benevolência &, 225 \\
Ideologia de higiene mental &, 008 \\
Restrição social &, 226 \\
Etiologia interpessoal &, 005 \\
Etiologia de esforço mental &, 000 \\
Visão minoritária &, 000 \\
\hline
\end{tabular}

\section{Discussão}

No fator Autoritarismo, a mediana 10,0 em relação ao fator Sten e os dados da Tabela 1 indicam que a grande maioria da população entrevistada apresenta fortes tendências a ser autoritária referindo que o paciente com transtorno mental deve ser isolado de outras pessoas, pois não há recuperabilidade. $\mathrm{O}$ escore de 1,24 no fator Benevolência, mediana 1,00, corrobora para uma postura autoritária, pois quanto menos benevolente tende a ser o indivíduo, maior a sua tendência ao autoritarismo. Comparando os escores do presente estudo a um estudo com enfermeiros de serviços psiquiátricos, nota-se que os enfermeiros estudados são mais autoritários e menos benevolentes do que os enfermeiros de serviços psiquiátricos ${ }^{(5)}$.

Observa-se na Tabela 1 que os fatores Benevolência e Ideologia de higiene mental foram os fatores com os escores abaixo da média descrita em seu estudo de validação ${ }^{(9)}$ e os demais fatores foram, de forma geral, bem acima da média esperada. Essa característica qualifica a concepção sobre doença mental dos profissionais dos serviços de urgência como extremamente autoritária, pouco benevolente e pouco consciente dos fatores que desencadeiam o transtorno mental. Quanto aos fatores acima da média esperada, permite caracterizar a concepção dos profissionais que atuam nos serviços de urgência como preconceituosa, ao mesmo tempo que indica o pouco conhecimento científico sobre os transtornos mentais.

Na Tabela 2, o teste de Kruskall-Wallis não apontou diferenças estatisticamente significantes entre auxiliar administrativo, enfermeiro, técnico 
de enfermagem e médico em relação aos fatores da escala de ODM, nos fatores Autoritarismo, Benevolência e Restrição Social ( $\mathrm{p}=0,894$, $p=0,225$ e $p=0,226$ respectivamente), fazendo que se aceite a hipótese nula para o teste, com relação a esses fatores, mas não descartando a presença de tal associação em uma amostra maior de estudo. Para os demais fatores, há diferenças estatisticamente significativas $(p<0.05)$.

Já no fator Ideologia de Higiene Mental para enfermeiros, a mediana de 4,00 permanece levemente abaixo do quantil 50 em relação ao fator Sten, significando aceitação abaixo da média de que o doente mental é semelhante às pessoas ditas normais, mas diferente do encontrado em outro estudo realizado com enfermeiros em dois hospitais que obtiveram fatores Sten de 6 e $7^{(5)}$.

Pelo Teste de Kruskall-Wallis, na Tabela 2, houve diferença estatisticamente significativa entre as quatro profissões analisadas em relação à Ideologia de Higiene Mental $(\mathrm{p}=0,008)$, Etiologia Interpessoal $(p=0,005)$, Etiologia de Esforço Mental $(\mathrm{p}=0,000)$ e Visão Minoritária ( $\mathrm{p}=0,000)$, ou seja, as quatro profissões têm pontos de vista distintos sobre o doente mental nesses fatores, afirmando o pressuposto na hipótese do presente estudo.

No fator Restrição Social, as respostas indicaram uma crença total de que esses pacientes apresentam transtornos hereditários, devendo permanecer no convívio de sua família (mediana $=10)$. No entanto, os escores foram inferiores aos encontrados nos estudos em 3 hospitais analisados, que variaram de 3,5 a 5,5 com uma amostra apenas de enfermeiros ${ }^{(5)}$. Isso revela uma noção de periculosidade mais arraigada nesses enfermeiros do que em relação aos enfermeiros de serviços psiquiátricos ${ }^{(5)}$.

Segundo a Tabela 1, para as profissões de saúde, no fator Restrição Social, obteve-se a mediana 10,00 para os técnicos de enfermagem, enfermeiros e médicos, obtendo-se um escore de 9,82, 9,94 e 9,86 no fator Sten. No entanto, segundo a Tabela 2, o teste de Kruskall-Wallis não apontou diferenças estatisticamente significantes entre as quatro profissões $(p=0,226)$.

Em relação à Etiologia Interpessoal, os sujeitos acreditam fortemente que os transtornos mentais são relacionados a fatores interpessoais ligados a graus de parentesco (mediana $=9,0$ ).
Contudo, outro estudo ${ }^{(5)}$ mostra escores de fator Sten médios menores ou iguais a 4,6 em que os enfermeiros nas quatro instituições analisadas neste estudo menos acreditam que os transtornos desses pacientes são originários das vivências interpessoais.

Em Etiologia de Esforço Mental indica uma crença total dos profissionais na crença de que essa doença é provinda de esforço mental exacerbado (mediana=10). Achado discrepante ${ }^{(5)}$, ao apresentado em outro estudo, em que não se identificou a crença de que clientes desenvolveram transtornos mentais por excessivo esforço mental.

Pelo Teste de Kruskall-Wallis, Tabela 2, houve diferença estatisticamente significativa entre as quatro profissões em relação aos fatores da escala Ideologia de Higiene Mental $(p=0,008)$, Etiologia interpessoal $(p=0,005)$, Etiologia de Esforço Mental $(p=0,000)$ e Visão Minoritária $(\mathrm{p}=0,000)$, ou seja, as quatro profissões têm pontos de vista distintos sobre o doente mental, influenciados, provavelmente, por suas profissões e formações acadêmicas.

Com relação à Visão Minoritária, acreditam que os pacientes são diferentes devido à sua aparência, diferenciando-os dos ditos normais (mediana $=7$ ), sendo refutada ${ }^{(5)}$, em que os enfermeiros em estudo apresentaram índices baixos em relação a esse fator, menor ou igual a 3,7. Pelo Teste de Kruskall-Wallis, Tabela 2, houve diferença estatisticamente significativa entre as quatro profissões $(p=0,000)$, ou seja, as quatro profissões têm pontos de vista distintos sobre o doente mental.

\section{Considerações Finais}

Com exceção aos fatores Benevolência e Ideologia de higiene mental, os demais fatores apresentaram escores elevados, bem acima da média identificada no estudo de validação da escala ODM. Para os enfermeiros, conclui-se que estes possuem uma crença mais estereotipada do que os enfermeiros de serviços de saúde mental em relação à doença e ao doente mental.

Pensando-se sobre as categorias profissionais estudadas, as diferenças encontradas nos quatro fatores da escala ODM foram importantes para descrever o tipo de diferença existente na concepção 
sobre doença mental, uma vez que, até o presente momento, se pressupunha diferenças, mas não se tinha conhecimento da magnitude destas. Nesse aspecto, o estudo traz contribuições importantes para o tema por ser um estudo pioneiro.

O teste de normalidade demonstrou que apenas o enfermeiro possui uma distribuição normal em sua amostra para os fatores Ideologia de Higiene Mental e Visão Minoritária, ou seja, a concepção sobre doença mental para os enfermeiros, nesses dois fatores, tende a apresentar esse mesmo padrão em quantidades maiores de sujeitos com essas características estudadas.

Considerou-se como limitação neste estudo o tamanho da amostra, podendo levar-nos ao possível erro tipo I, condicionado às possibilidades financeiras de concretização da pesquisa, assim como também a inexistência de pesquisas envolvendo as outras categorias profissionais estudadas, limitando a análise dos dados. Salientase, ainda, a importância de outros estudos, bem como o investimento em ações de educação continuada para todas as categorias profissionais envolvidas, principalmente aos enfermeiros, uma vez que a escala utilizada sugere uma atitude não desejável, permeada de preconceito e estigma, diante do doente mental.

\section{Referências}

1. Gonçalves AM, Sena RR. Psychiatric reform in Brazil: contextualization and consequences regarding the care for the mentally ill in their family environment. Rev. Latino-Am. Enfermagem. 2001;9:48-55.

2. Scherer ZAP, Scherer EA, Labate RC. Psychiatric nursing consultation and liaison: how do nurses understand this activity? Rev. Latino-Am. Enfermagem. 2002;10:7-14.

3. Del-Ben CM, Rufino AC, Azevedo-Marques JM, Menezes PR. Differential diagnosis of firstepisode psychosis: importance of optimal approach in psychiatric emergencies. Rev Bras Psiquiatr. [Internet]. 2010;32(Suppl 2)S78-86. [acesso 2 nov 2011]. Disponível em: http://www.scielo. br/scielo.php? script $=$ sci_arttext\&pid $=$ S 1516$-44462010000600004 \& \operatorname{lng}=$ en. http://dx.doi. org/10.1590/S1516-44462010000600004.

4. Souza AMA, Braga VAB. Brazilian psychiatric reform: a lot to reflect on. Acta
Paul. Enferm. [Internet]. 2006 [acesso 2 nov 2011]. Disponível em: http://www.scielo.br/ scielo.php?script $=$ sci_arttext\&pid $=$ S0103$-21002006000200013 \& \operatorname{lng}=$ en. http://dx.doi. org/10.1590/S0103-21002006000200013.

5. Tavares RR, Pedrão LJ. Comparative analysis of nurses' views from different psychiatric units on an individual in psychic suffering] Cogitare Enferm. 2003;8:75-86.

6. Pedrão LJ, Avanci RC, Malaguti SE, Aguilera AMS. Atitudes frente à doença mental: estudo comparativo entre ingressantes e formandos em enfermagem. Medicina. (Ribeirão Preto). 2003;36(1):37-44.

7. Delevati DM, Palazzo LS. Businessmen attitudes regarding mental disordered individuals in the south of Brazil. J Bras Psiquiatria. 2008;57:240-6.

8. Arvaniti A, Samakouri M, Kalamara E, Bochtsou V, Bikos C, Livaditis M. Health service staff's attitudes towards patients with mental illness. Soc Psychiatry Psychiatr Epidemiol. 2009;44(8):658-65.

9. Rodrigues CRC. Atitudes frente à doença mental: estudo transversal de uma amostra de profissionais da saúde. [Tese de doutorado]. Ribeirão Preto: Faculdade de Medicina de Ribeirão Preto da Universidade de São Paulo; 1983.

10. Cohen J, Struening EL. Opinions about mental illness in the personnel of two large mental hospitals. J Abnorm Socy Psychol. 1962;64:349-60. 11. Canfield AA. The sten scale: a modified C. scale. Educ Psychol Measure. 1951;11:295-7.

12. Struening EL, Cohen J. Fatorial invariance and other psychometric characteristics of five opinions about mental illness fators. Educ Psychol Measure. 1963;23:289-98. 